\title{
Access to alcohol and binge drinking among vocational college students: A multilevel study in a tourist destination province of Thailand
}

\author{
Edward McNeil $^{1}$, Yanisa Inthawong ${ }^{2}$, and Sawitri Assanangkornchai ${ }^{1}$ \\ ${ }^{1}$ Epidemiology Unit, Faculty of Medicine, Prince of Songkla University, Hat Yai, Songkhla 90112, Thailand \\ ${ }^{2}$ Primary Health Care Unit, Thalang Hospital, Thalang, Phuket 83110, Thailand
}

\begin{abstract}
A key issue in alcohol-related harm reduction is the impact of commercial and social availability on alcohol-related problems and harm among young people. The increasing density of alcohol outlets has been shown to be associated with harmful youth drinking behavior, although studies have produced mixed results, underlying the complexity of the situation.
\end{abstract}

Aims: The present study was conducted to assess the relationship between density of alcohol outlets and drinking behaviors among vocational college students.

Methods: A cross-sectional school-based survey was conducted among full-time students studying in vocational colleges in Phuket, Thailand. Multilevel regression models were used to assess the relationship between alcohol-outlet density and current and binge drinking, controlling for student and school characteristics.

Results: A total of 3,363 students completed the self-reported questionnaire (response rate 66.7\%). A significant association was found between alcohol-outlet density and binge drinking but not current drinking. Both current and binge drinking were associated with a positive attitude toward drinking, perception of peer and family drinking norms, and social availability of alcohol. For every increase in 10 on-premise alcohol outlets per square kilometer the risk of binge drinking increased by an average of 5\%. Empirical evidence regarding this relationship is important to support law and policy movements towards further restriction of alcohol outlets and zoning of entertainment venues.

Efforts to reduce alcohol-related harm in recent years have focused on the perceptions and drinking practices of young people and identifying and responding to known factors that encourage high-risk drinking (Lipperman-Kreda, Grube, \& Paschall, 2010; Toomey et al., 2006).

A key issue in alcohol-related harm reduction is the impact of point-of-sale and other means of physical availability on alcohol-related problems and harm among young people. In addition to having negative consequences at the individual level, the increasing number of alcohol outlets in communities may have a negative impact on family members due to alcohol-related violence and crime (Cunradi, Mair, Ponicki, \& Remer, 2011; Jennings et al., 2014; Lipton et al., 2013). A study in New Zealand found an association between binge drinking and the density of off-license liquor outlets within easy walking distance of home, with a $4 \%$ increase in the odds of binge drinking for every additional outlet (Connor, Kypri, Bell, \& Cousins,
2011). The density of alcohol outlets has also been identified as a potential source of influence for alcohol consumption (Scribner et al., 2008), harm (Kavanagh et al., 2011), and violence (Grubesic, Pridemore, Williams, \& Philip-Tabb, 2013). A positive association between alcohol-outlet density and alcohol consumption was found among college students (Weitzman, Folkman, Folkman, \& Wechsler, 2003), while off-premise outlet density was found to be associated with both perceived and actual informal and formal access to alcohol (Treno, Ponicki, Remer, \& Gruenewald, 2008). Other studies found the density of alcohol outlets to be correlated with individual drinking (Kypri, Bell, Hay, \& Baxter, 2008), quantities consumed among teenage drinkers (Huckle, Huakau, Sweetsur, Huisman, \& Casswell, 2008), and perceived availability and volume of drinking among adolescents (Kuntsche, Kuendig, \& Gmel, 2008). Furthermore, the density of packaged liquor outlets was found to be significantly associated with very high-risk drinking in

Correspondence: Sawitri Assanangkornchai, MD, PhD, FRCPsychT, Epidemiology Unit, Faculty of Medicine, Prince of Songkla University , 15 Kanchanavanich Road, Hat Yai, Songkhla 90110, Thailand. Telephone: +6674451165, Fax: +6674429754; E-mail: savitree.a@psu.ac.th

Financial support: This study was supported by the Center for Alcohol Studies of the Thai Health Promotion Foundation. The Epidemiology Unit, where EM and SA work, is partially supported by the National Science and Technology Development Agency, Ministry of Science and Technology. The funding sources had no involvement in study design, data collection, analysis and interpretation of data, the writing of the report, or the decision to submit the article for publication. Declaration of interest: None

Keywords: alcohol outlets, binge drinking, vocational students, Thailand 
Australia (Livingston, Laslett, \& Dietze, 2008). A more recent study found a positive association between onpremise outlet density with heavy episodic drinking among young Swiss men in the southwest part of Switzerland (Astudillo, Kuendig, Centeno-Gil, Wicki, \& Gmel, 2014). In contrast, a U.S.-based study found no relationship between alcohol outlet exposure by distance and density around resident's home and alcohol use (Pollack, Cubbin, Ahn, \& Winkleby, 2005), and a study in New Zealand found no association between alcohol-outlet density and average alcohol consumption or risky drinking, although outlet density was positively associated with binge drinking (Connor et al., 2011). Overall, it appears that research to date has produced mixed results on the relationship between outlet density and alcohol consumption and harm, with most studies being conducted in developed countries among college students.

One of the effective strategies recommended globally to prevent alcohol-related harm is to regulate the commercial or public availability of alcohol through laws and policies in accordance with cultural norms. This measure includes the regulation of the number and location of on- and offpremise alcohol outlets (World Health Organization, 2010). Given the mixed findings related to the relationship between alcohol-outlet density and consumption among youth stated above, and the complexity of this relationship, it would be interesting to know if this relationship exists in a context where underage drinking is socially and legally unacceptable but availability of alcohol is high. Being a fast-growing commercial hub in Southeast Asia, Thailand has gone through a lot of changes in its culture recently (McGregor, 2008). Globalization has also had a significant effect on the lifestyle of its people, including drinking behaviors. The prevalence of alcohol use has been increasing continuously, and alcohol abuse among young people is one of the main public health concerns of the Thai government.

A Thai national survey in 2007 showed that among youths aged $12-19$ years, $17.9 \%$ were current drinkers and $6.1 \%$ had alcohol use disorders (Assanangkornchai, Sam-Angsri, Rerngpongpan, \& Lertnakorn, 2010). A national school survey in 2007 found that vocational students had the highest rate of alcohol consumption and tended to be involved more with frequent binge and intoxicated drinking than high-school students (Assanangkornchai, Pattanasattayawong, Samangsri, \& Mukthong, 2007). Binge drinkers were significantly more likely to have drinking consequences (e.g., driving after drinking, nausea and vomiting, and having a hangover) than were non-binge drinkers (Assanangkornchai, Mukthong, \& Intanont, 2009).

A more recent study suggests that the rates of alcohol consumption among high-school students may have plateaued (Tantirangsee, Assanangkornchai, \& Geater, 2014). In response to the high impact of youth drinking, several public policies and social movements have been launched in Thailand in the past decade, including a limit on the physical availability of alcohol. Some of the policies include the control of the number of alcohol outlets and zoning of entertainment and drinking venues in some areas. Under the Alcohol Beverage Control Act (Alcoholic Beverage Control Laws, 2008), the legal age for purchasing alcohol was raised from 18 to 20 years and alcohol trading hours were restricted to 11 a.m. -2 p.m. and 5 p.m.-12 a.m. Alcohol sales and consumption are also prohibited in educational areas and religious institutions. Empirical evidence is needed to support this law. The present study was thus conducted to determine whether alcohol-outlet density has any relationship with alcohol consumption among students attending vocational colleges in Phuket, a tourist destination province in southern Thailand.

\section{Methods}

\section{Study Design and Sample}

A cross-sectional, school-based survey was conducted between July and September 2011 in Phuket province of southern Thailand. Phuket province is administratively divided into three districts: Muang, Thalang, and Kathu. Each of the three districts is further divided into subdistricts, comprising altogether 17 subdistricts. The local population in 2012 was approximately 350,000, with a population density of 605 persons per square kilometer (Phuket Provincial Public Health Office, 2012). There are six vocational schools in the province; five are situated in Muang district, which is the central business area of the province, while the other one is a public school located in Thalang district, a semi-rural district (Figure 1).

All full-time students studying in all of the six vocational colleges of the province were invited to participate in the study. Students were informed about the objective of the study and were given the right to refuse without having any effect on their study. The project was approved by the Ethics Committee for Research in Human Subjects of the Faculty of Medicine, Prince of Songkla University.

\section{Measures}

A self-reported, anonymous paper-based questionnaire was used to collect student data. Data collection was conducted during a regular class period with permission given from the class teachers and took between 15-20 minutes to complete. The questionnaire was modified from the one used in the series of repeated national school surveys of alcohol consumption and other health-risk behaviors (Assanangkornchai et al., 2009). It contains five sections: socio-demographic characteristics, such as age, sex, living situation (alone, with family, or with friends), parents' income, and work status; school performance, as measured by grade point average (GPA; range $0-4.00$ ); perception of peer and family drinking norms; student's mobility; student's access to alcohol; and frequency and pattern of alcohol consumption. Peer and family drinking norms were measured by asking students the number of friends and family members who drank, the percentage of friends who drank, and whether drinking was acceptable among their friends and family members. The student's mobility was measured by asking whether the student or any of the student's closest friends had a car or motorcycle. Positive 
Figure 1

Distribution of alcohol outlet density (outlets $/ \mathrm{km}^{2}$ ) in Phuket province, Thailand

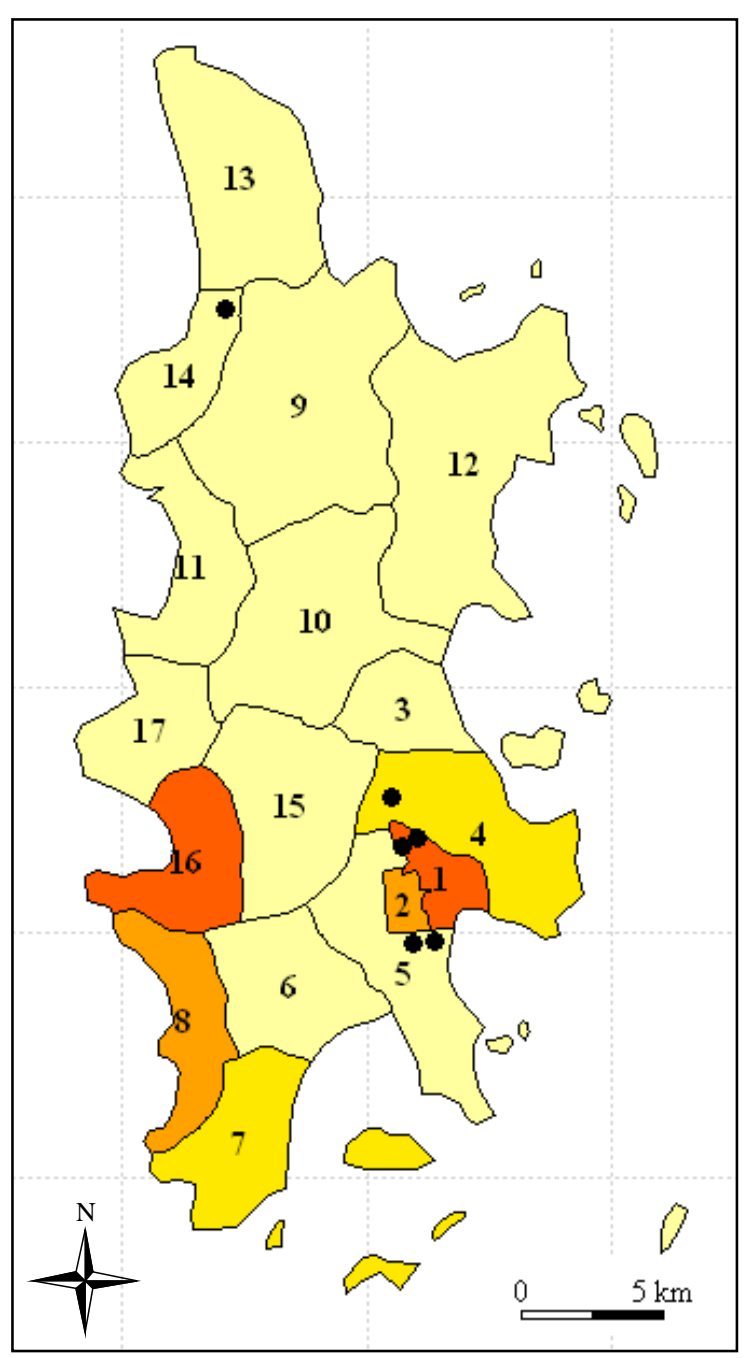

attitudes towards alcohol drinking were indicated by 13 yes-no questions (e.g., drinking enhances social skills; drinking enhances stress- or problem-coping skills; drinking increases appetite; and drinking increases the chance of having sex). A positive attitude to each question was given a score of 1 , and the average score of each student was obtained. Access to alcohol was measured by asking students where they usually obtained their alcohol (outlets, parents, older adults) and whether the outlet from which they obtained alcohol usually asked for proof of age. Pattern of alcohol consumption included questions on frequency and quantity of alcohol intake. Current drinking was defined as consumption of at least one standard drink of an alcoholic beverage (a 330-ml can of beer, a 100-ml glass of wine, or a 30-ml glass of whiskey or spirits) in the past 30 days. Prevalence of current drinking is reported as the percentage among lifetime drinkers as well as overall. Heavy episodic drinking or binge drinking was defined for males as the consumption of at least five alcoholic



Alcohol outlet density per $\mathrm{km}^{2}$

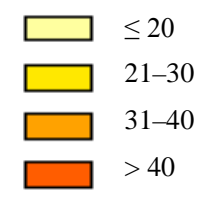

beverages on a single 2-hour drinking occasion in the past 30 days; for females, it was defined as the consumption of at least four alcoholic beverages over the same time period. Prevalence of binge drinking is reported as the percentage among current drinkers as well as overall.

School data were obtained from the school administrators, including type (private/public), number of students, average socio-economic status of the students' families, and school policies relating to alcohol consumption.

All alcohol outlets in the province were identified using a walk-through survey conducted by the research team, who were familiar with the area. Geographic locations of the outlets were collected by global positioning system receivers. Types of alcohol outlets were also identified and classified as either off- or on-premise. An off-premise alcohol outlet was defined as a place where alcoholic beverages are sold for consumption elsewhere-for 
example, liquor shops, supermarkets, convenience stores, grocery stores, and kiosks. An on-premise outlet was defined as a venue where alcoholic beverages are sold for consumption on the site of sale-for example, pubs, bars, and restaurants. The alcohol-outlet density in the student's neighborhood was defined as the number of outlets per 100 square meters of the subdistrict where the student lived. Phuket is inhabited by long-stay tourists and itinerant workers; thus the number of alcohol outlets per geographical area is a more valid measure of density than number per population, and this definition was chosen for this study.

Previous studies have shown that several factors predict youths' drinking behavior, including youths' perceptions of community norms and parental approval of drinking, perceived alcohol availability, alcohol use by friends and siblings, social source of alcohol, and ease of access to alcohol (Kuntsche et al., 2008; Lipperman-Kreda et al., 2010). Thus, all of these factors were taken into account in considering the effect of alcohol-outlet density on the students' drinking.

\section{Statistical Analyses}

All statistical analyses were conducted using the $\mathrm{R}$ language and environment ( $\mathrm{R}$ Development Core Team, 2011). The two main outcome variables were current and binge drinking. The main exposure variable, alcohol-outlet density, was measured as the number of all types of alcohol outlets, off-premise or on-premise, per square kilometer of the subdistrict.

The sampling weights for a one-stage survey design were calculated by multiplying the total number of students in the school divided by the number of sampled students in that school. Multi-level mixed-effects logistic regression models were fit to the data, adjusting for individual and school variables. The individual-level exposure variables were student's socio-demographic characteristics, GPA, perceptions of peer and family drinking norms, and student's mobility, while community-level variables were school characteristics and alcohol-outlet density. As the minimal legal age for purchasing alcohol is 20 years in Thailand, age was categorized into $\geq 20$ or $<20$ years to reflect this status. Other demographic characteristics were gender, school level, living situation, work status, and subdistrict of residence. The modeling strategy began by choosing variables with $p$ values $<.2$ from the univariate analysis and including them in the multivariate mixedeffects models. Backward elimination was used to retain or remove the variables from each model step based on the Akaike Information Criterion. Variables with a $p$ value $<.05$ were included in the final model. Adjusted odds ratios (AOR) and 95\% confidence intervals (CI) were presented for variables selected in the final models.

\section{Results}

\section{Sample Characteristics}

Of 5,022 registered students in the six vocational schools in the 2011 academic year, 3,363 students completed the questionnaire, giving a response rate of $66.7 \%$ (range 52.5$84.4 \%$ per school). The main reasons for non-response were absenteeism and internship outside school at the time of data collection. Among the responders, 1,683 were male (50.0\%) and 1,668 were female (49.6\%), while 12 did not identify their gender. The mean age of students was 16.9 years (standard deviation $=1.61$ years, range $14-26$ ), with $87 \%$ being aged under 20 years. Most students (67\%) were not concurrently working, and $84 \%$ still lived with their parent(s) or other member(s) of their family. The mean GPA was 2.74 ( \pm 0.52), indicating a moderate level of school performance. The numbers of students in the study who lived in each of the 17 subdistricts ranged from 40 to 525.

\section{Patterns of Drinking}

Table 1 shows patterns of drinking stratified by gender. About half of the students reported having drunk alcohol at some time in their lives, with a higher percentage in males (64.8\%) than in females (42.5\%). Among 1,789 lifetime drinkers, 607 were current drinkers (drank at least once in the past 30 days), with males having a slightly higher prevalence. Among these 607 current drinkers, most drank between 1 and 2 days in total within the past-30-day period, with an average of 1.4 days in males and 1.5 days in females. More than one third of the students drank 1-2 standard drinks per drinking day on average, while $44.2 \%$ of current drinkers reported binge drinking. The overall prevalence rates of current and binge drinking were $16.8 \%$ (males 22.6\%, females 11.3\%) and 7.3\% (males 10.8\%, females $3.2 \%$ ), respectively.

\section{Characteristics of Alcohol Outlets and Prevalence of Drinking}

A total of 8,026 operating alcohol outlets were identified, of which 4,341 were located in Muang district, 1,677 were located in Thalang district, and 2,008 were located in Kathu districts. Among these, 3,864 (48.1\%) were off-premise outlets and 4,162 (51.9\%) were on-premise outlets. There were 14 different types of alcohol-selling venues identified in Phuket. On-premise venues included beer gardens, entertainment complexes, hotels, karaoke lounges, pubs/bars, restaurants, and snooker clubs; off-premise venues included convenience stores, drug stores, grocery stores, kiosks, liquor stores, and supermarkets. The three most common types of outlets identified were grocery stores (48.3\%), restaurants (21.4\%), and public bars (10.0\%). Among the 17 subdistricts, the average number of outlets per square kilometer was approximately 14 (range 2-218). Talat Yai, the central business area of Phuket, had the highest density of outlets (218 per square kilometer), followed by Patong, a beachside tourist attraction (86 per square kilometer). The prevalence of current drinking of the students of each subdistrict ranged from $9 \%$ to $23 \%$, while the prevalence of binge drinking varied from less than $1 \%$ to $10 \%$ (Table 2 ). 
Table 1

Weighted prevalence of drinking by gender

\begin{tabular}{|c|c|c|c|c|}
\hline Variable & $\begin{array}{c}\text { Male } \\
\%(S E)\end{array}$ & $\begin{array}{l}\text { Female } \\
\%(S E)\end{array}$ & $\begin{array}{c}\text { Total } \\
\%(S E)\end{array}$ & $n$ \\
\hline Lifetime drinker & $64.8(1.80)$ & $42.5(1.98)$ & $51.9(1.60)$ & 1,789 \\
\hline \multicolumn{5}{|c|}{ Drinking status among lifetime drinkers $\left(n=1,782^{*}\right)$} \\
\hline Former drinker & $29.8(2.52)$ & $38.2(2.97)$ & $32.8(2.36)$ & 588 \\
\hline Past-year drinker & $35.2(3.07)$ & $35.2(2.50)$ & $34.9(2.50)$ & 587 \\
\hline Current drinker & $35.0(2.58)$ & $26.6(1.70)$ & $32.3(2.18)$ & 607 \\
\hline \multicolumn{5}{|c|}{ Frequency of current drinking (drinking in the past 30 days) $\left(n=535^{*}\right)$} \\
\hline $1-2$ days & $57.8(4.65)$ & $76.6(2.26)$ & $61.4(3.87)$ & 347 \\
\hline$\geq 3$ days & $42.2(4.65)$ & $23.4(2.63)$ & $38.6(3.87)$ & 188 \\
\hline \multicolumn{5}{|c|}{ Quantity of current drinking (average drinks/day) $(n=595 *)$} \\
\hline 1-2 standard drinks & $35.4(3.12)$ & $53.8(0.52)$ & $39.9(2.76)$ & 264 \\
\hline $3-4^{\dagger}$ standard drinks & $15.8(1.76)$ & $17.9(1.93)$ & 15.8 (1.38) & 105 \\
\hline$\geq 5^{\ddagger}$ standard drinks & $48.8(0.41)$ & $28.3(2.28)$ & $44.2(3.48)$ & 226 \\
\hline
\end{tabular}

Note: SE, standard error. * Missing values excluded. ${ }^{\dagger} 3$ standard drinks for females. ${ }^{\ddagger} \geq 4$ standard drinks for females.

Table 2

Prevalence of current and binge drinking and alcohol outlet density in Phuket, Thailand, by subdistrict

\begin{tabular}{lcccccccc}
\hline & \multicolumn{1}{c}{ Subdistrict* } \\
\cline { 2 - 9 } & $\mathbf{1}$ & $\mathbf{2}$ & $\mathbf{3}$ & $\mathbf{4}$ & $\mathbf{5}$ & $\mathbf{6}$ & $\mathbf{7}$ & $\mathbf{8}$ \\
\hline Prevalence of current drinking & 0.20 & 0.20 & 0.18 & 0.19 & 0.19 & 0.20 & 0.22 & 0.20 \\
Prevalence of binge drinking & 0.09 & 0.10 & 0.02 & 0.07 & 0.06 & 0.10 & 0.10 & 0.10 \\
Alcohol outlets & & & & & & & & \\
$\quad$ On-premise (n) & 466 & 115 & 32 & 362 & 190 & 167 & 306 & 530 \\
$\quad$ Off-premise (n) & 404 & 167 & 83 & 610 & 307 & 254 & 238 & 113 \\
Density per km & 217.5 & 35.3 & 2.4 & 27.3 & 8.9 & 14.0 & 22.9 & 30.6 \\
\hline
\end{tabular}

*Subdistrict codes: 1. Talat Yai, 2. Talat Nuea, 3. Koh Kaew, 4. Ratsada, 5. Wichit, 6. Chalong, 7. Rawai, 8. Karon,.

\begin{tabular}{lccccccccc}
\hline & \multicolumn{1}{c}{ Subdistrict* } \\
\cline { 2 - 11 } & $\mathbf{9}$ & $\mathbf{1 0}$ & $\mathbf{1 1}$ & $\mathbf{1 2}$ & $\mathbf{1 3}$ & $\mathbf{1 4}$ & $\mathbf{1 5}$ & $\mathbf{1 6}$ & $\mathbf{1 7}$ \\
\hline Prevalence of current drinking & 0.10 & 0.14 & 0.09 & 0.11 & 0.13 & 0.15 & 0.23 & 0.17 & 0.15 \\
Prevalence of binge drinking & 0.02 & 0.06 & 0.03 & 0.01 & 0.08 & 0.03 & 0.09 & 0.07 & 0.01 \\
Alcohol outlets & & & & & & & & & \\
$\quad$ On-premise (n) & 100 & 74 & 191 & 27 & 90 & 66 & 87 & 1178 & 181 \\
$\quad$ Off-premise (n) & 217 & 184 & 229 & 202 & 189 & 108 & 214 & 228 & 120 \\
Density per km & 4.0 & 7.8 & 10.2 & 6.0 & 9.6 & 8.8 & 9.5 & 85.7 & 15.9 \\
\hline
\end{tabular}

*Subdistrict codes: 9. Thep Krasattri, 10. Si Sunthon, 11. Cheng Thale, 12. Pa Khlok, 13. Mai Khao, 14. Sakhu, 15. Kathu, 16. Patong, 17. Kamala.

\section{Predictors of Current Drinking}

Table 3 shows statistically significant predictors from the multilevel modeling. Individual-level factors significantly associated with being a current drinker were not living with family members, having friend(s) or siblings(s) who drank, having positive attitudes towards drinking, and having more than $40 \%$ of close friends who drank. Moreover, students who reportedly could obtain alcohol from older adults or from an alcohol outlet without requiring to show 
proof of age were more likely to drink alcohol in the past 30 days compared to those who reported otherwise. Other individual-level variables collected were not statistically significant in the multivariate analysis. At the community level, alcohol-outlet density was not significantly associated with current drinking. School-level variables were also not associated with current drinking.

\section{Predictors of Binge Drinking}

Individual-level predictors for binge drinking were similar to those for current drinking except that living situation was not significant. Having friends who drank regularly or having more than $40 \%$ of close friends who drank alcohol increased the risk of being a binge drinker by 7.9 and 4.7 times, respectively, compared to those who had not. No other individual-level variable was statistically significant in the multivariate analysis. At the community level, alcohol-outlet density was significantly associated with binge drinking. Density per 10 square kilometers of onpremise outlets was significantly associated with binge drinking $(\mathrm{AOR}=1.05,95 \% \mathrm{CI}=1.01-1.09$ ). Thus, for every increase in 10 on-premise alcohol outlets per square kilometer, the risk of binge drinking increased by $5 \%$. Density of off-premise drinking outlet was not statistically significant in the final model.

\section{Table 3}

Prevalence of current and binge drinking, adjusted odds ratios, and 95\% confidence intervals for significant individual-level and community-level factors.

\begin{tabular}{|c|c|c|c|c|}
\hline & \multicolumn{2}{|c|}{ Current drinking } & \multicolumn{2}{|c|}{ Binge drinking } \\
\hline & $\%^{a}$ & $\operatorname{AOR}(95 \% \mathrm{CI})^{\mathrm{b}}$ & $\%^{\mathrm{a}}$ & AOR $(95 \% \text { CI })^{b}$ \\
\hline \multicolumn{5}{|l|}{ Individual-level factors } \\
\hline Living situation: (ref = With family) & 34.0 & & 6.6 & \\
\hline Alone or with friend(s) & 45.1 & $1.97(1.28-3.05)$ & 8.2 & \\
\hline Sibling drinks alcohol: (ref = No) & 28.0 & & 4.0 & \\
\hline Yes & 55.9 & $1.40(1.08-1.81)$ & 15.3 & $2.22(1.55-3.19)$ \\
\hline Friends drink alcohol: (ref = Never) & 11.6 & & 0.8 & \\
\hline Rarely & 53.7 & $2.43(1.87-3.16)$ & 8.9 & $2.16(1.50-3.11)$ \\
\hline Regularly & 61.4 & $2.23(1.40-3.56)$ & 32.2 & $7.24(3.47-15.1)$ \\
\hline Obtains alcohol from adults: (ref = No) & 54.7 & & 7.0 & \\
\hline Yes & 73.2 & $2.00(1.55-2.57)$ & 17.2 & $2.26(1.46-3.49)$ \\
\hline Alcohol outlet asks for proof of age: (ref = Yes) & 57.0 & & 5.4 & \\
\hline No & 77.5 & $1.89(1.44-2.48)$ & 23.1 & $2.96(1.96-4.46)$ \\
\hline Positive attitude toward drinking (mean) & 0.59 & $1.61(1.16-2.23)$ & 0.67 & $2.54(1.53-4.23)$ \\
\hline Percent of close friends who drink: $($ ref $\leq 20)$ & 20.8 & & 1.1 & \\
\hline $21-40$ & 29.4 & $1.12(0.83-1.49)$ & 4.0 & $1.89(0.94-3.79)$ \\
\hline $41-60$ & 46.7 & $1.52(1.10-2.12)$ & 7.4 & $3.14(1.54-6.37)$ \\
\hline $61-80$ & 57.4 & $2.17(1.44-3.27)$ & 16.4 & $4.81(2.39-9.68)$ \\
\hline$>80$ & 66.2 & $2.26(1.49-3.42)$ & 26.4 & $6.51(3.30-12.9)$ \\
\hline \multicolumn{5}{|l|}{ Community level factors } \\
\hline On-premise density ${ }^{\mathrm{c}}\left(10\right.$ outlets $/ \mathrm{km}^{2}$ ) & & $1.02(0.99-1.05)$ & & $1.05(1.01-1.09)$ \\
\hline Off-premise density ${ }^{\mathrm{c}}\left(10\right.$ outlets $/ \mathrm{km}^{2}$ ) & & $1.02(0.98-1.05)$ & & $1.05(0.99-1.10)$ \\
\hline
\end{tabular}

AOR, adjusted odds ratio; CI, confidence interval.

${ }^{\mathrm{a}}$ Unweighted row percentage

${ }^{\mathrm{b}}$ Odds ratios adjusted for all other variables in the model, including on-premise outlet density, compared to non-drinkers of the same time frame.

${ }^{\mathrm{c}}$ Odds ratios for on-premise and off-premise outlet density variables were calculated from running separate models.

\section{Discussion}

This study found an association between binge drinking and the density of alcohol outlets within home neighborhoods independent of students' individual factors, with a $5 \%$ increase in the odds of binge drinking for every increase in 10 on-premise alcohol outlets per square kilometer. Several studies of college students have found a significant positive relationship between binge drinking and outlet density (Chaloupka, Grossman, \& Saffer, 2002; Kypri et al., 2008; Weitzman et al., 2003), but this seems to 
be the first study in vocational students of a developing country with high controls relating to underage drinking. The increase in binge drinking in high alcohol-outlet density areas is an important public health concern. If this association represents a causal relationship, then the effect of restricting outlet density on binge drinking would be extensive. Public health policy advocators in Thailand, as in many other countries, are currently developing strategies to reduce underage drinking. This study confirms that the density of outlets is a legitimate public health concern. The number of alcohol outlets has been increasing substantially in many areas across the country over the past decade, with a growth in the number of on-premise outlets and attractive marketing practices such as sales prices, promotions, and advertisements, which appear to be associated with youth drinking (Kuo, Wechsler, Greenberg, \& Lee, 2003). The regulation regarding restriction of on-premise outlet density may be an important strategy and useful public health tool for reducing excessive binge drinking.

The differential association for on- and off-premise outlets and binge drinking may be explained by the nature of drinking by this young group of people. Binge drinking is a common pattern of alcohol consumption among youths (Miller, Naimi, Brewer, \& Jones, 2007). Students of this age group tend to drink for fun with their friends in a gathering place near their place of residence where they can easily access alcoholic beverages with a motorcycle, the most popular means of transportation in Phuket. Therefore, having a large number of on-premise drinking venues in their home neighborhood serves their purpose well. In contrast, density of off-premise alcohol outlets had no association with both current drinking and binge drinking. Previous studies have also found that on- and off-premise outlets are differently related to drinking behaviors (Astudillo et al., 2014; Connor et al., 2011; Kypri et al., 2008). The difference in drinking cultures and population characteristics may be the reason underlying the discrepancy across studies.

The current study shows that individual factors were associated with students' current drinking. This is consistent with other studies showing that students who were living with someone other than their own parents, who had friends or family members who drank alcohol (Assanangkornchai et al., 2009), or who were able to obtain alcohol from home or social sources (Chen, Gruenewald, \& Remer, 2009; Hearst, Fulkerson, Maldonado-Molina, Perry, \& Komro, 2007; Shamblen et al., 2011) were significantly more likely to drink and have access to alcohol. Prevention efforts should target students' attitudes and perceived community norms towards drinking. Having friends or siblings who drink, or perceiving that drinking is acceptable by parents and friends, may create normative beliefs among youths that drinking is socially accepted. More importantly, most underage people obtain alcohol through social sources or acquaintances (Chen, Grube, \& Gruenewald, 2010; Hearst et al., 2007) or venues that illegally sell alcohol to apparent minors (Freisthler, Gruenewald, Treno, \& Lee, 2003; Toomey et al., 2004). Our results thus suggest that communities need to control social sources of alcohol-for example, through enforcement of social provision laws, development of monitoring systems, and focusing on alcohol-establishment behavior, such as asking for proof of age before selling alcohol (Toomey, Lenk, \& Wagenaar, 2007)—to reduce the supply of alcoholic beverages to youths.

These study findings should be considered in light of the following limitations. First, the cross-sectional design of the study limits the ability to make causal inferences. Second, the estimated $66.7 \%$ response rate at the individual level may have undermined the ability to generalize the findings to other non-participating school-attending youths. In this instance, bias might have been present if the response rate was associated with alcohol drinking. Third, although the survey questionnaire was anonymous, the students' responses might have been subject to recall bias. The findings might also have been affected by reporting bias and under-reporting of alcohol use stemming from legal concerns of underaged students. However, there is no reason to believe that a student in a particular subdistrict under-reported his or her alcohol use any differently than a student in another subdistrict. Thus, although the overall results may be an underestimation of the true alcohol consumption situation of students in Phuket province, the relationship between alcohol-outlet density and alcohol consumption found in this study should still be valid. Finally, the effect of a student's neighboring subdistrict was not taken into account in the modeling. It is possible that students living on the border of a subdistrict may be more likely to frequent licensed premises in a neighboring subdistrict if it is closer than another one in the student's own subdistrict. However, this is unlikely to occur in the Thai context, since communities in bordering subdistricts are quite remote from each other.

Nevertheless, we have used an accurate technique to measure the density of alcohol outlets and differentiated between types of outlets, thus enriching the current literature. We have identified additional policy levers for preventing binge drinking among youths. Two obvious policy options are to encourage a shift in the cultural perception of underage drinking, perhaps through reductions in alcohol advertisements, which has been shown to increase the likelihood that adolescents drink (Anderson, de Bruijn, Angus, Gordon, \& Hastings, 2009; Smith \& Foxcroft, 2009), and an increase in law enforcement of existing regulations that limit underage purchasing and consumption of alcohol. A third option is to restrict the number of alcohol outlets, especially the onpremise outlets. As stated before, evidence does suggest that reductions in availability of alcohol outlets can have significant impacts on drinking behavior and negative alcohol-related consequences (Astudillo et al., 2014; Huckle et al., 2008; Kuntsche et al., 2008; Kypri et al., 2008; Livingston et al., 2008). Other approaches could be to reduce the availability of social sources of alcohol, to put more control on the sale behavior, and to modify drinking norms. A recent review (Cisneros Örnberg \& Room, 2014) concluded that heavy drinking among tourists, encouraged by increased access and availability of alcohol, had substantial impact on alcohol consumption of locals, particularly young people. In light of this and our own 
results, policy makers must strike a balance between minimizing the harm from binge drinking on local youths and maximizing returns from the sale of alcohol to tourists. Phuket relies heavily on tourism and it will be a challenge for the government to satisfy local business interests while at the same time protecting precious local cultures and traditional values. One recent Thai study has shown that residents can still maintain their local cultures and traditions under the threat of globalization and influx of Western tourists (Ukrit, Arunotai, \& Doungchan, 2011). In summary, this study indicates that alcohol-outlet density has a significant relationship with harmful drinking behaviors among Thai vocational students, regardless of their age and sex.

\section{References}

Alcoholic Beverage Control Laws, 125 C. F. R. (2008). In National Legislation Council (ed.), The Royal Decree (Vol. 125, pp. 34-48). Bangkok, Thailand: Author.

Anderson, P., de Bruijn, A., Angus, K., Gordon, R., \& Hastings, G. (2009). Impact of alcohol advertising and media exposure on adolescent alcohol use: A systematic review of longitudinal studies. Alcohol and Alcoholism, 44, 229-243.

Assanangkornchai, S., Mukthong, A., \& Intanont, T. (2009). Prevalence and patterns of alcohol consumption and health-risk behaviors among high school students in Thailand. Alcoholism, Clinical and Experimental Research, 33(12), 2037-2046.

Assanangkornchai, S., Pattanasattayawong, U., Samangsri, N., \& Mukthong, A. (2007). Substance use among high-school students in southern Thailand: Trends over 3 years (2002-2004). Drug and Alcohol Dependence, 86(2-3), 167-174.

Assanangkornchai S., Sam-Angsri N., Rerngpongpan S., \& Lertnakorn A. (2010). Patterns of alcohol consumption in the Thai population: Results of the National Household Survey of 2007. Alcohol and Alcoholism. 45(3), 278-285.

Astudillo, M., Kuendig, H., Centeno-Gil, A., Wicki, M., \& Gmel, G. (2014). Regional abundance of on-premise outlets and drinking patterns among Swiss young men: District level analyses and geographic adjustments. Drug and Alcohol Review, 33(5), 526-533.

Chaloupka, F. J., Grossman, M., \& Saffer, H. (2002). The effects of price on alcohol consumption and alcoholrelated problems. Alcohol Research \& Health, 26(1), 22-34.

Chen, M. J., Grube, J. W., \& Gruenewald, P. J. (2010). Community alcohol outlet density and underage drinking. Addiction, 105(2), 270-278.

Chen, M. J., Gruenewald, P. J., \& Remer, L. G. (2009). Does alcohol outlet density affect youth access to alcohol? Journal of Adolescent Health, 44(6), 582589.

Cisneros Örnberg, J., \& Room, R. (2014). Impacts of tourism on drinking and alcohol policy in low and middle-income countries: A selective thematic review. Contemporary Drug Problems, 41, 145-169.
Connor, J. L., Kypri, K., Bell, M. L., \& Cousins, K. (2011). Alcohol outlet density, levels of drinking and alcoholrelated harm in New Zealand: A national study. Journal of Epidemiology \& Community Health, 65(10), 841-846.

Cunradi, C. B., Mair, C., Ponicki, W., \& Remer, L. (2011). Alcohol outlets, neighborhood characteristics, and intimate partner violence: Ecological analysis of a California city. Journal of Urban Health, 88(2), 191200.

Freisthler, B., Gruenewald, P. J., Treno, A. J., \& Lee, J. (2003). Evaluating alcohol access and the alcohol environment in neighborhood areas. Alcoholism: Clinical \& Experimental Research, 27(3), 477-484.

Grubesic, T. H., Pridemore, W. A., Williams, D. A., \& Philip-Tabb, L. (2013). Alcohol outlet density and violence: The role of risky retailers and alcoholrelated expenditures. Alcohol and Alcoholism, 48(5), 613-619.

Hearst, M. O., Fulkerson, J. A., Maldonado-Molina, M. M., Perry, C. L., \& Komro, K. A. (2007). Who needs liquor stores when parents will do? The importance of social sources of alcohol among young urban teens. Preventive Medicine, 44(6), 471-476.

Huckle, T., Huakau, J., Sweetsur, P., Huisman, O., \& Casswell, S. (2008). Density of alcohol outlets and teenage drinking: Living in an alcogenic environment is associated with higher consumption in a metropolitan setting. Addiction, 103(10), 1614-1621.

Jennings, J. M., Milam, A. J., Greiner, A., Furr-Holden, C. D., Curriero, F. C., \& Thornton, R. J. (2014). Neighborhood alcohol outlets and the association with violent crime in one mid-Atlantic city: The implications for zoning policy. Journal of Urban Health, 91(1), 62-71.

Kavanagh, A. M., Kelly, M. T., Krnjacki, L., Thornton, L., Jolley, D., Subramanian, S. V., . . . Bentley, R. J. (2011). Access to alcohol outlets and harmful alcohol consumption: A multi-level study in Melbourne, Australia. Addiction, 106(10), 1772-1779.

Kuntsche, E., Kuendig, H., \& Gmel, G. (2008). Alcohol outlet density, perceived availability and adolescent alcohol use: A multilevel structural equation model. Journal of Epidemiology \& Community Health, 62(9), 811-816.

Kuo, M., Wechsler, H., Greenberg, P., \& Lee, H. (2003). The marketing of alcohol to college students: The role of low prices and special promotions. American Journal of Preventive Medicine, 25(3), 204-211.

Kypri, K., Bell, M. L., Hay, G. C., \& Baxter, J. (2008). Alcohol outlet density and university student drinking: A national study. Addiction, 103(7), 1131-1138.

Lipperman-Kreda, S., Grube, J. W., \& Paschall, M. J. (2010). Community norms, enforcement of minimum legal drinking age laws, personal beliefs and underage drinking: An explanatory model. Journal of Community Health, 35(3), 249-257.

Lipton, R., Yang, X., Braga, A. A., Goldstick, J., Newton, M., \& Rura, M. (2013). The geography of violence, alcohol outlets, and drug arrests in Boston. American Journal of Public Health, 103(4), 657-664. 
Livingston, M., Laslett, A. M., \& Dietze, P. (2008). Individual and community correlates of young people's high-risk drinking in Victoria, Australia. Drug and Alcohol Dependence, 98(3), 241-248.

McGregor, J. A. (2008). Wellbeing, development and social Change in Thailand. Thammasat Economic Journal, 26(2), 1-27.

Miller, J. W., Naimi, T. S., Brewer, R. D., \& Jones, S. E. (2007). Binge drinking and associated health risk behaviors among high school students. Pediatrics, 119(1), 76-85.

Phuket Provincial Public Health Office. (2012). Phuket population. Bangkok, Thailand: National Statistical Office. Retrieved from http://www.pkto.moph.go.th/

Pollack, C. E., Cubbin, C., Ahn, D., \& Winkleby, M. (2005). Neighbourhood deprivation and alcohol consumption: Does the availability of alcohol play a role? International Journal of Epidemiology, 34(4), 772-780.

R Development Core Team. (2011). R: A language and environment for statistical computing. Vienna, Austria: R Foundation for Statistical Computing.

Scribner, R., Mason, K., Theall, K., Simonsen, N., Schneider, S. K., Towvim, L. G., \& DeJong, W. (2008). The contextual role of alcohol outlet density in college drinking. Journal of Studies on Alcohol and Drugs, 69(1), 112-120.

Shamblen, S. R., Harris, M. S., Ringwalt, C. L., Flewelling, R. L., Clark, H. K., \& Hanley, S. M. (2011). Outlet density as a predictor of alcohol use in early adolescence. Substance Use \& Misuse, 46(8), 10491059.

Smith, L., \& Foxcroft, D. (2009). The effect of alcohol advertising, marketing and portrayal on drinking behaviour in young people: Systematic review of prospective cohort studies. BMC Public Health, 9, 51.

Tantirangsee, N., Assanangkornchai, S., \& Geater, A. F. (2014). Trends and associated factors of alcohol consumption among Southern Thai adolescents, 20032009. International Journal of Alcohol and Drug Research, 3(3), 219-225.

Toomey, T. L., Fabian, L. A., Erickson, D. J., Wagenaar, A. C., Fletcher, L., \& Lenk, K. M. (2006). Influencing alcohol control policies and practices at community festivals. Journal of Drug Education, 36(1), 15-32.

Toomey, T. L., Lenk, K. M., \& Wagenaar, A. C. (2007). Environmental policies to reduce college drinking: An update of research findings. Journal of Studies on Alcohol and Drugs, 68(2), 208-219.

Toomey, T. L., Wagenaar, A. C., Erickson, D. J., Fletcher, L. A., Patrek, W., \& Lenk, K. M. (2004). Illegal alcohol sales to obviously intoxicated patrons at licensed establishments. Alcoholism: Clinical and Experimental Research, 28(5), 769-774.

Treno, A. J., Ponicki, W. R., Remer, L. G., \& Gruenewald, P. J. (2008). Alcohol outlets, youth drinking, and selfreported ease of access to alcohol: A constraints and opportunities approach. Alcoholism: Clinical and Experimental Research, 32(8), 1372-1379.

Ukrit, A., Arunotai, N., \& Doungchan, P. (2011). The dynamics and protection of local culture under globalization on Lanta Island in Southern Thailand. Journal of Contextual Behavior Science, 7(5), 23-28.

Weitzman, E. R., Folkman, A., Folkman, M. P., \& Wechsler, H. (2003). The relationship of alcohol outlet density to heavy and frequent drinking and drinkingrelated problems among college students at eight universities. Health \& Place, 9(1), 1-6.

World Health Organization. (2010). Global strategy to reduce the harmful use of alcohol. Geneva, Switzerland: Author. 